\title{
Scientific Knowledge Graph of Acupuncture for Migraine: A Bibliometric Analysis from 2000 to 2019
}

\author{
Yanqing Zhao $\mathbb{D}^{1, *}$ \\ Li Huang ${ }^{2} *$ \\ Meijuan Liu' \\ Han Gao' \\ Wentao $\mathrm{Li}^{1,3}$
}

'Internal Medicine of Traditional Chinese Medicine, Shanghai Municipal Hospital of Traditional Chinese Medicine, Shanghai, People's Republic of China; ${ }^{2}$ Internal Medicine of Traditional Chinese Medicine, Putuo Hospital, Shanghai University of Traditional Chinese Medicine, Shanghai, People's Republic of China; ${ }^{3}$ Encephalopathy Department, Shanghai Municipal Hospital of Traditional Chinese Medicine, Shanghai University of Traditional Chinese Medicine, Shanghai,

People's Republic of China

*These authors contributed equally to this work
Correspondence: Wentao Li

Encephalopathy Department, Shanghai Municipal Hospital of Traditional Chinese Medicine, 274 Zhijiang Middle Road, Jingan District, Shanghai, 20007I, People's Republic of China

Tel +86 I33 31882730

Email IwtII32@I63.com
Objective: This study aims to explore the trend and knowledge mapping of acupuncture for migraine through bibliometrics.

Methods: It retrieved the literature on acupuncture for migraine in the Web of Science database from 2000 to 2019, and then resorted to CiteSpace to conduct bibliometric analysis to attain the knowledge mapping.

Results: The total number of publications each year has increased year by year, and the average annual growth rate from 2000 to 2009 was $15.57 \%$, while from 2010 to 2019 was $6.35 \%$, with a faster growth rate from 2000 to 2009 . According to the cluster analysis of institutions, authors, cited references, and keywords, 10, 7, 12, and 10 categories were gained from 2000 to 2019. The most productive countries, institutions, and authors are the USA and China, Technical University of Munich and Beijing University of Chinese Medicine, Linde $\mathrm{K}$ and Liang FR from 2000 to 2019, whose frequency is $119 / 103,28 / 24$, and $28 / 24$, respectively. However, the most important of them are Canada, Sichuan University, and Witt CM. Owing to their highest centrality, they are $0.86,0.54$, and 0.27 separately. Moreover, cited references that contributed to the most co-citations are Linde K (2005), yet, the most vital cited reference is Karst M (2001). Keywords such as migraine, acupuncture, headache, pain, and randomized controlled trial are the most frequently used. However, needle acupuncture is the crucial keyword. In the cluster analysis of institutions, authors, cited references, and keywords from 2000 to 2019, the largest cluster categories are \#0 migraine prophylaxis, \#1 randomized controlled trial, \#0 episodic migraine, and \#0 topiramate treatment. Then, randomized controlled trials of acupuncture prevention and treatment of migraine are the most important research content in this field.

Conclusion: Through the bibliometric analysis of the research on acupuncture for migraine in the Web of Science database in the past 20 years, the trends and the Knowledge Graph of the country, institution, author, cited reference, and the keyword are acquired, which have an important guiding significance for quickly and accurately positioning the key information in the field.

Keywords: acupuncture, migraine, scientific knowledge graph, CiteSpace, bibliometric analysis

\section{Introduction}

Migraine is a group of recurrent headache diseases, showing unilateral or bilateral pain, often accompanied by nausea and vomiting. A few typical cases have visual, sensory, and motor disorders and other auras before the attack, ${ }^{1}$ and may have a family history. ${ }^{2}$ According to a study of 2016 global burden of disease study, 1.04 billion people worldwide suffer from migraines, with an overall prevalence rate of $14.4 \%{ }^{3}$ 
Migraine affects women three times as much as men, ${ }^{4}$ the prevalence of migraine in children increases with age. ${ }^{5}$ And the annual prevalence of migraines in the elderly is about $10 \%{ }^{6}$ Migraine is the main cause of disability in the world, the number of years of disability-adjusted life lost caused by migraine has reached one third. ${ }^{7}$ With the increase of headache days, the disease burden (disability, medical care utilization, and direct cost) of migraine patients also increases. ${ }^{8}$ The high incidence rate, high disability rate, and the increase of treatment expenditure have brought serious economic costs and brought various burdens to society. It is vital to quickly and accurately grasp the research trends of migraines. And the drug treatment of migraine includes acute drug therapy and intermittent preventive drug therapy. Presently, the treatment in the acute phase mainly utilizes non-steroidal anti-inflammatory drugs, barbiturates, opioids, and triptans. ${ }^{9}$ Although the above-mentioned drugs have achieved positive effects in the treatment and prevention of migraines, long-term use has more side effects. Such as longterm use of NSAIDs may cause gastrointestinal reactions, bleeding risks, liver and kidney damage, and headaches caused by drug overuse; ergotamine long-term high-dose medication can see muscle pain, precordial pain, the sudden withdrawal of drugs can appear rebound headache, also easy to produce psychological dependence. ${ }^{10,11}$ Given that migraines may be chronic and therefore, require long-term control, current research should explore treatment options that focus on high efficiency and minimal side effects. ${ }^{12}$ This has led many clinicians and researchers to turn to other forms of treatment, such as acupuncture, as an alternative source of pain management and healing for patients with refractory migraines with standard medications. ${ }^{13}$ Traditional Chinese medicine treatment (including acupuncture) has the characteristics of "overall regulation", which has advantages in reducing the attack frequency of migraine, relieving headache symptoms, and improving the quality of life. $^{14}$

Bibliometrics is an interdisciplinary science that uses mathematical and statistical methods to quantitatively analyze all knowledge carriers. It uses statistical indicators to measure the contribution to a research field, ${ }^{15}$ including different countries, institutions, journals, or authors and predicts trends or hotspots in a field. ${ }^{16}$ The research methods involved include co-word analysis and cluster analysis. Co-word analysis is an important method of bibliometrics. ${ }^{17}$ It can be used to identify trends and hotspots. The number of occurrences in the group of literature, this number of co-occurrences to measure the close relationship between them. Cluster analysis utilizes a weighting algorithm, log-likelihood algorithm, and mutual information algorithm with "title entries", "index entries", and "abstract entries" to extract common information to interpret research mainstreams and research characteristics. $^{18}$ Researchers rely on relevant analysis software for visual analysis (including Citespace, ${ }^{19}$ VOSviewer, ${ }^{20}$ Histcite, $^{21}$ etc.).

Up to now, there has been no comprehensive article of studies on acupuncture for migraine using bibliometric methods. Therefore, in this study, to analyze the global status and trend of acupuncture for migraine in the past 20 years, the researchers utilized the bibliometric software CiteSpace to not only analyze the growth of publications in this field from 2000 to 2019 of the Web of Science Database (SCI-E) but also construct the knowledge maps of countries, institutions, authors, cited references and keywords from 2000 to 2019, which is of great significance to accurately grasp the research trends and hotspots.

\section{Methods}

\section{Source of Literature}

To prevent the omission of searching the literature, we obtained the synonyms for "migraine" and "acupuncture" through the MeSH Database in PubMed, and then amalgamate the final data. Foremost, we input the Web of Science database with English Topic= "migraine", and TS= "acupuncture" OR “Acupuncture Therapy” OR “Acupuncture, Ear" OR “Acupuncture Points" OR "Acupuncture Analgesia" separately. ${ }^{22}$ Next is the parameter setting when retrieving the database, kinds of literature were retrieved online through the Science Citation IndexExpanded of the Web of Science Core Collection on March 1, 2021 23 , and the language of the literature is not limited.The time range of searching is from 2000 to 2019, which is the basis of data analysis. After literature retrieval, a total of 749 kinds of literature were acquired, and 438 records were utilized for bibliometric analysis after Citespace removed duplication. Data removal is done in the form of a combination of manual verification and CiteSpace software. And the searched Web of science database stems from the Tsing Hua University Library database in China.

\section{Analysis Software}

The version of visual software is Citespace 5.2.R1, which was invented by Professor Chen Chaomei from the 
Computer and Information Science of Drexel University in the United States, and can be used to analyze the structure, laws, and distribution of scientific knowledge. ${ }^{24}$

\section{Parameter Setting}

Time slicing (from 2000 to 2019, years per slice: 5), node type (check one at a time, including Author, Institution, Country, Keyword, Cited Reference), pruning select Pathfinder, Pruning sliced networks, and Pruning the merged network.

\section{Statistical methods}

Bibliometric analysis was implemented on all acupuncture for migraine literature. Frequency was the main metric used to identify the core countries/territories, institutions, authors, cited references, and keywords. Centrality means betweenness centrality, which is an indicator to measure the importance of nodes in the network. Citespace uses this indicator to discover and measure the significance of kinds of literature and utilizes purple circles to highlight such literature. Pieces of literature with high betweenness centrality are usually the key hub connecting two different fields. It is also called a turning point in Citespace. This method of calculating the importance of nodes is proposed by Freeman in 1977. Betweenness centrality Calculated as follows:

$$
B C_{i}=\sum_{s \neq i \neq t} \frac{n_{s t}^{i}}{g_{s t}}
$$

In the formula, $g_{s t}$ is the number of shortest paths from node $\mathrm{s}$ to node $\mathrm{t}$, and $n_{s t}^{i}$ is the number of shortest paths through node $\mathrm{i}$ among $g_{s t}$ shortest paths from node $\mathrm{s}$ to node $t$. From the perspective of information transmission, the higher the betweenness centrality, the greater the importance of the node. The result of clustering analysis is a keyword co-occurrence network. The cluster view emerges the distribution of fields from a different point of view. The timeline view primarily reveals solicitude for delineating the relationship between clustering results and concentrates on the historical span of literature in a clustering result. CiteSpace offers the module value (called $\mathrm{Q}$ value) and the silhouette value (called $\mathrm{S}$ value) to judge the effect of map drawing. The $Q$ value is generally in the interval $[0,1]$, and $Q>0.3$ implies that the allocated community structure is conspicuous. When the $\mathrm{S}>0.7$, the clustering is the most reliable; if $\mathrm{S}>0.5$, clustering is generally rational. ${ }^{25}$

\section{Research Ethics}

These data are downloaded from the Web of Science database; these are secondary data and do not involve interactions with animals or humans.

\section{Results and Discussion Analysis of the Total Number of Publications}

In order to find the development trend of the total number of publications accurately, researchers divided it into two periods (2000-2009 and 2010-2019) ${ }^{26}$ for comparative analysis, In Figure 1, we found an interesting phenomenon: Comparing the average total number of publications of the two-time nodes, it is found that the number of publications in 2000-2009 is 15.9 and that in 2010-2019 is 28.0. The number of outputs in the second period is higher than that in the first period. However, the growth rate of the two periods is reckoned by using the growth rate calculation formula (growthrate $=\left(\frac{\text { present }}{\text { past }}\right)^{\bar{n}}-1, \mathrm{n}$ is the number of years), which is $15.57 \%$ in $2000-2009$ (The total number of publications in 2000 was 8 , and that in 2009 was 34) and $6.35 \%$ in 2010-2019 (The total number of publications in 2010 was 20, and that in 2009 was 37). It can also be further confirmed that there is a smooth transition period from 2013 to 2016, and the total number of publications fluctuates a little. Therefore, we can think that the growth rate of publications in 2000-2009 is greater than in 2010-2019, and the overall trend is to increase year by year (the total growth rate is $16.55 \%$ ).

\section{Analysis of Countries/Territories/ Institutions}

A network map with 24 nodes and 19 links of countries/ territories was created (Figure 2), and a cluster map (Modularity $\mathrm{Q}=0.8278$, Silhouette $=0.6373$ ) with 130 nodes and 158 links of institutions was engendered, along with a timeline view. The countries/territories/institutions that published papers on acupuncture for migraine in 2000-2019 are revealed in Tables 1 and 2. The top 5 countries/territories are the USA, Peoples R China, Germany, England, and Italy, and the most prolific institutions were Tech Univ Munich, followed by Beijing Univ Chinese Med, Chengdu Univ Tradit Chinese Med, Capital Med Univ, and Mem Sloan Kettering Canc Ctr. However, the value of centrality reflects the importance of countries/ territories/institutions in the node of the cooperative relationship network, so the most important countries/ 


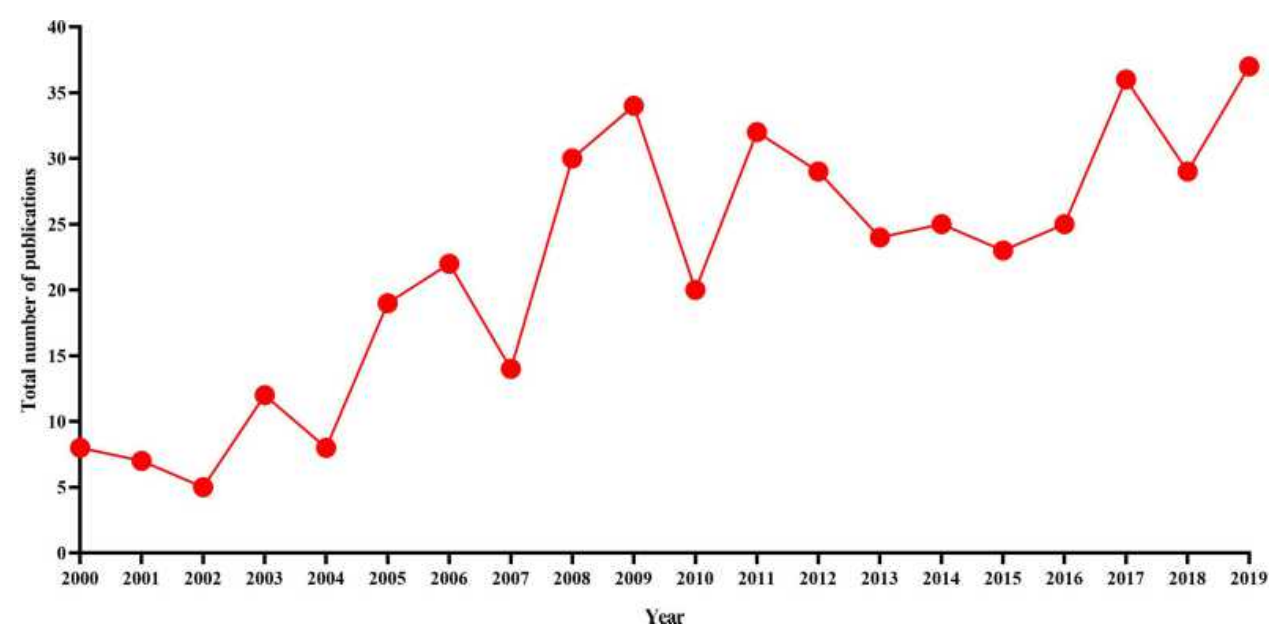

Figure I Line Chart of Yearly Output on acupuncture for Migraine. The abscissa in the figure represents the year and the ordinate represents the total number of publications.

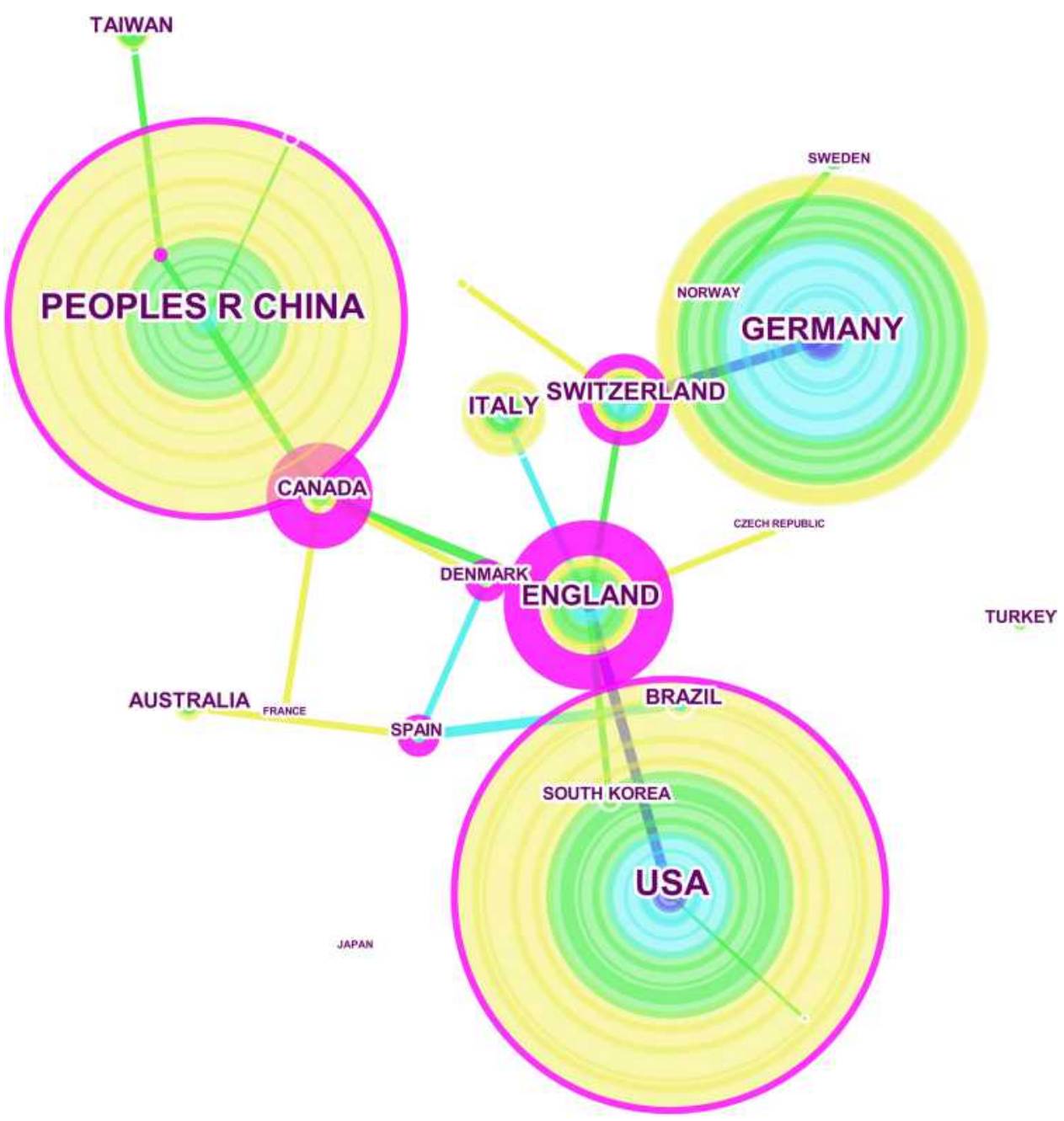

Figure 2 Network of countries/territories on acupuncture for Migraine. The purple node in the middle of the annual ring means the influence and the significance of a country/territory. The larger the node and the more purple it exhibits, the greater is the importance of the country/territory. 
Table I Countries/ Territories Contributed to Publications on Acupuncture for Migraine from 2000 to 2019

\begin{tabular}{|l|c|c|c|}
\hline $\begin{array}{l}\text { Countries/ } \\
\text { Territories }\end{array}$ & Frequency & $\begin{array}{c}\text { Countries/ } \\
\text { Territories }\end{array}$ & Centrality \\
\hline USA & 119 & Canada & 0.86 \\
\hline Peoples R China & 103 & England & 0.82 \\
\hline Germany & 90 & Denmark & 0.36 \\
\hline England & 41 & Switzerland & 0.26 \\
\hline Italy & 32 & Spain & 0.26 \\
\hline Switzerland & 25 & USA & 0.13 \\
\hline Brazil & 16 & Peoples R China & 0.13 \\
\hline Canada & 15 & Scotland & 0.13 \\
\hline Australia & 14 & Germany & 0.00 \\
\hline Taiwan (China) & 14 & Italy & 0.00 \\
\hline Spain & 11 & Brazil & 0.00 \\
\hline South Korea & 10 & Australia & 0.00 \\
\hline Turkey & 8 & Taiwan (China) & 0.00 \\
\hline Denmark & 8 & South Korea & 0.00 \\
\hline Sweden & 6 & Turkey & 0.00 \\
\hline Note: In ths & 140 & coluns repres & \\
\hline
\end{tabular}

Note: In this table, the other two identical columns represent different rankings. The left column of the table represents the Countries/ Territories that have published acupuncture for migraine. The frequency of contribution is sorted from high to low. The column on the right represents the sorting from high to low according to centrality.

institutions are Canada (0.86) and Sichuan Univ (centrality $=0.54$ ), followed by England (0.82), Denmark (0.36), Switzerland (0.26), and Spain (0.26), and the following institutions are Univ York (0.49), Chengdu Univ Tradit Chinese Med (0.44), Hunan Univ Tradit Chinese Med (0.36) and Mem Sloan Kettering Canc Ctr (0.34).

Through the above analysis, we found an interesting phenomenon: from the outputs of acupuncture for migraine, China ranks second, second only to the USA, but from the perspective of centrality, China's importance in this field is weaker than that of the Western developed countries and other developing countries have fewer outputs and the cooperation between countries is not close enough, which is also its limitation. Besides, we also discovered that most institutions engaged in acupuncture for migraine are from China, with the University of Traditional Chinese Medicine as the main body of research, there are two reasons for this phenomenon,
Table 2 Institutions Contributed to Publications on Acupuncture for Migraine from 2000 to 2019

\begin{tabular}{|c|c|c|c|}
\hline Institution & Frequency & Institution & Centrality \\
\hline Tech Univ Munich & 28 & Sichuan Univ & 0.54 \\
\hline $\begin{array}{l}\text { Beijing Univ } \\
\text { Chinese Med }\end{array}$ & 24 & Univ York & 0.49 \\
\hline $\begin{array}{l}\text { Chengdu Univ } \\
\text { Tradit Chinese } \\
\text { Med }\end{array}$ & 23 & $\begin{array}{c}\text { Chengdu Univ } \\
\text { Tradit Chinese } \\
\text { Med }\end{array}$ & 0.44 \\
\hline Capital Med Univ & 19 & $\begin{array}{c}\text { Hunan Univ Tradit } \\
\text { Chinese Med }\end{array}$ & 0.36 \\
\hline $\begin{array}{l}\text { Mem Sloan } \\
\text { Kettering Canc } \\
\text { Ctr }\end{array}$ & 15 & $\begin{array}{c}\text { Mem Sloan } \\
\text { Kettering Canc } \\
\text { Ctr }\end{array}$ & 0.34 \\
\hline Univ Turin & 12 & Peking Univ & 0.32 \\
\hline $\begin{array}{l}\text { China Acad } \\
\text { Chinese Med Sci }\end{array}$ & 12 & $\begin{array}{c}\text { Shandong Univ } \\
\text { Tradit Chinese } \\
\text { Med }\end{array}$ & 0.31 \\
\hline Univ York & 11 & Aalborg Univ & 0.19 \\
\hline Sichuan Univ & 11 & $\begin{array}{l}\text { Hunan Univ } \\
\text { Chinese Med }\end{array}$ & 0.17 \\
\hline Univ Maryland & II & Univ Maryland & 0.15 \\
\hline Harvard Univ & 10 & Univ Oxford & 0.15 \\
\hline Univ N Carolina & 10 & Univ Ottawa & 0.15 \\
\hline $\begin{array}{l}\text { Ruhr Univ } \\
\text { Bochum }\end{array}$ & 10 & $\begin{array}{l}\text { Oregon Coll } \\
\text { Oriental Med }\end{array}$ & 0.15 \\
\hline Xidian Univ & 8 & $\begin{array}{c}\text { China Acad } \\
\text { Chinese Med Sci }\end{array}$ & 0.12 \\
\hline Charite & 8 & $\begin{array}{c}\text { Massachusetts } \\
\text { Gen Hosp }\end{array}$ & 0.12 \\
\hline
\end{tabular}

Note: In this table, the other two identical columns represent different rankings. The left column of the table represents the institutions that have published acupuncture for migraines. The frequency of contribution is sorted from high to low. The column on the right represents the sorting from high to low according to centrality.

Abbreviations: Tech Univ Munich, Technical University of Munich; Beijing Univ Chinese Med, Beijing University of Chinese Medicine; Chengdu Univ Tradit Chinese Med, Chengdu University of Traditional Chinese Medicine; Capital Med Univ, Capital medical university; Mem Sloan Kettering Canc Ctr,Memorial SloanKettering Cancer Center; Univ Turin, University of Turin; China Acad Chinese Med Sci,China Academy of Chinese Medical Sciences; Univ York, University of York; Sichuan Univ, Sichuan University; Univ Maryland, University of Maryland; Harvard Univ, Harvard University; Univ N Carolina, University of North Carolina; Ruhr Univ Bochum, Ruhr University Bochum; Xidian Univ, Xidian University; Hunan Univ Tradit Chinese Med, Hunan University of Traditional Chinese Medicine; Peking Univ, Peking University; Shandong Univ Tradit Chinese Med, Shandong University of Traditional Chinese Medicine; Aalborg Univ, Aalborg University; Hunan Univ Chinese Med, Hunan University of Chinese Medicine; Univ Oxford, University of Oxford; Univ Ottawa, University of Ottawa; Oregon Coll Oriental Med,Oregon College of Oriental Medicine; Massachusetts Gen Hosp, Massachusetts General Hospital. 

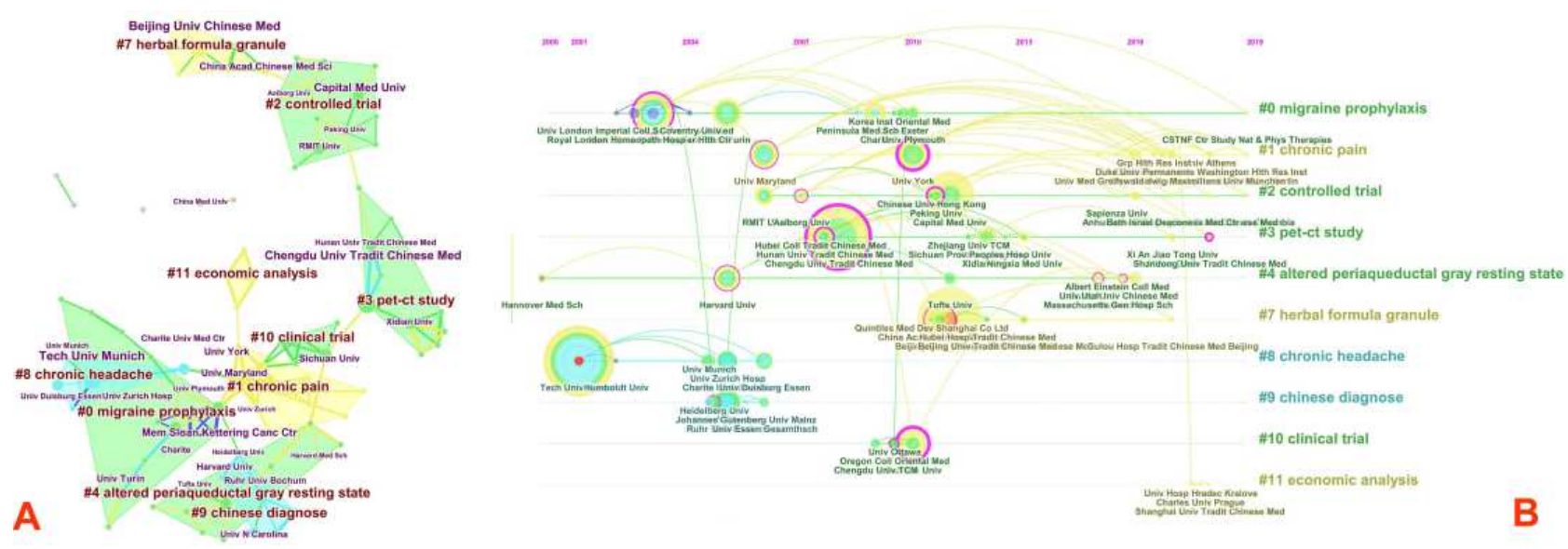

Figure 3 (A) Cluster map of institutions based on label clusters with title terms. The combination of symbols and numbers delegates the institutions' study of similar categories. (B) Timeline zone of institutions on acupuncture for Migraine The purple node in the middle of the annual ring means the influence and the significance of the institution. Institutions with the homologous research category are on the same time horizon.

First, acupuncture originated in China; Second, China's government policies support higher education institutions more than other social institutions.

By means of cluster analysis of institutions (Figure 3A and Table 3), researchers can locate the same research category that different institutions are participated in. Figure $3 \mathrm{~B}$ is the timeline view of the co-citation analysis of institutions with the top 10 clusters, When the cluster view cannot estimate which institutions be absorbed in the same research in detail, it will make use of the cluster timeline view Figure $3 \mathrm{~B}$ to analyze. The whole modularity $\mathrm{Q}=0.8278>0.3$ corresponds to a significant community structure. And the largest cluster were \#0 migraine prophylaxis ( $\mathrm{S}$ value $=0.954>0.7$, Contains 14 institutions), $\# 1$ chronic pain ( $\mathrm{S}$ value $=0.865>0.7$, Contains 13 institutions), \#2 controlled trial ( $\mathrm{S}$ value $=0.954>0.7$, Contains 12 institutions), $\# 3$ pet-ct study ( $\mathrm{S}$ value $=0.848>0.7$, Contains 11 institutions). The relationship network in the category of migraine prophylaxis $(\# 0)$ is a cooperative network between Royal London Homeopath Hosp, Mem Sloan Kettering Canc Ctr, Univ Plymouth, and Univ Exeter. Furthermore, the earliest research in this direction can be traced back to Royal London Homeopath Hosp in 2002; The research direction is the relationship network of

Table 3 Institutions Engaged in Acupuncture for Migraine That Details of Knowledge Clusters

\begin{tabular}{|l|c|c|c|c|c|}
\hline Cluster ID & Size & Silhouette & Mean (Year) & Label (LLR) & Label (MI) \\
\hline 0 & 14 & 0.954 & 2007 & Migraine prophylaxis & Placebo effect \\
\hline 1 & 13 & 0.865 & 2015 & Contronic pain trial & Placebo effect \\
\hline 2 & 12 & 0.954 & 2012 & Pet-ct study & Placebo effect \\
\hline 3 & 11 & 0.848 & 2012 & Placebo effect \\
\hline 4 & 10 & 0.936 & 2012 & Altered periaqueductal gray resting state & Clinical trial \\
\hline 7 & 7 & 0.976 & 2012 & Herbal formula granule & Clinical trial \\
\hline 8 & 6 & 0.919 & 2004 & Chronic headache & Clinical trial \\
\hline 9 & 6 & 0.979 & 2005 & Chinese diagnose & Clinical trial \\
\hline 10 & 5 & 0.920 & 2009 & Clinical trial & Placebo effect \\
\hline 11 & 4 & 0.983 & 2018 & Economic analysis & Clinical trial \\
\hline
\end{tabular}

Note: The cluster analysis results mainly include cluster ID, mean year, size, silhouette, label (LLR), and label (MI). Cluster ID is the number after clustering, and Size represents the number of members contained in the cluster. The larger the Size is, the smaller the number. Mean Year represents the average year of the literature in the cluster, which can be used to judge the distance of the cited literature in the cluster. The larger the log-likelihood ratio (LLR) is, the more representative the cluster category; mutual information (MI) is mainly used to represent the relationship between terms and categories in text mining, and it does not consider the frequency of feature words. 
Table 4 Authors Contributed to Publications on Acupuncture for Migraine from 2000 to 2019

\begin{tabular}{|c|c|c|c|c|}
\hline No. & Author & Frequency & Author & Centrality \\
\hline 1 & Linde $\mathrm{K}$ & 28 & Witt CM & 0.27 \\
\hline 2 & Liang FR & 24 & Huang WJ & 0.23 \\
\hline 3 & Li Y & 18 & Willich SN & 0.19 \\
\hline 4 & Zheng $\mathrm{H}$ & 15 & Sun GJ & 0.17 \\
\hline 5 & Allais G & 15 & Lan L & 0.12 \\
\hline 6 & Diener HC & 15 & Allais G & 0.11 \\
\hline 7 & Witt CM & 14 & Melchart D & 0.11 \\
\hline 8 & Melchart D & 14 & Brinkhaus B & 0.11 \\
\hline 9 & Wang LP & 11 & $\mathrm{Li} Y$ & 0.08 \\
\hline 10 & Weidenhammer W & 11 & Vickers AJ & 0.07 \\
\hline II & Brinkhaus B & 11 & Zhao L & 0.07 \\
\hline 12 & MacPherson $\mathrm{H}$ & 10 & MacPherson $\mathrm{H}$ & 0.06 \\
\hline 13 & Willich SN & 9 & Zhang FW & 0.06 \\
\hline 14 & Liu CZ & 9 & Hammes $M$ & 0.05 \\
\hline 15 & Vickers AJ & 9 & Liu JX & 0.04 \\
\hline
\end{tabular}

Note: In this table, the other two identical columns represent different rankings. The left column of the table represents the authors that have published acupuncture for migraine. The frequency of contribution is sorted from high to low. The column on the right represents the sorting from high to low according to centrality.

chronic pain (\#1) that the cooperation network between Univ York, Univ Maryland, Nanjing Univ Chinese Med, Univ Southampton. In addition, the earliest research in this direction originated from Univ Maryland in 2006; The research direction of the institutional cooperation network of Capital Med Univ, Peking Univ, RMIT Univ, Anhui Univ Chinese Med, and Beijing Inst Tradit Chinese Med is controlled trial (\#2), of which the earliest research on this aspect appeared from RMIT Univ in 2006; The research orientation of the institutional cooperation network of Chengdu Univ Tradit Chinese Med, Hunan Univ Tradit Chinese Med, Zhejiang Univ TCM, Shandong Univ Tradit Chinese Med, Xidian Univ, Xi An Jiao Tong Univ is pet-ct study (\#3), and the earliest research on this aspect comes from Chengdu Univ Tradit Chinese Med in 2008.

It is beneficial for discovering similar researches between institutions and exploring potential cooperation relationships between them. However, the cooperation between institutions is mainly based on domestic universities in China, or the cooperation between international institutions is dominated, and the cooperation between international institutions is also mainly in developed countries. This has certain limitations for the development of acupuncture for migraines and we should try to overcome this disadvantage, enabling acupuncture to benefit people from all over the world.

\section{Analysis of Authors}

Considering the volume of published documents and centrality, the top 15 authors publishing articles were listed in Table 4. Linde $\mathrm{K}$ (Frequency=28) was identified as the most active author in the field, followed by Liang FR, Li $\mathrm{Y}$, Zheng $\mathrm{H}$, and Allais $\mathrm{G}$, and the authors with high centrality are Witt CM (0.27), Huang WJ, Willich SN, Sun GJ, and Lan L, therefore, they are considered to be the most important researcher in the Author's network relationship. Generating a cited author map resulted in 203 nodes and 398 links with a mean Silhouette, $\mathrm{S}=$ 0.6361 and Modularity $\mathrm{Q}, \mathrm{Q}=0.8845$ (Figure 4A). In this map, the modularity $\mathrm{Q}$ score was greater than 0.7 , which means the network was reasonably divided into loosely coupled clusters. Detailed information regarding the top 7 clusters and their timelines has been presented in Table 5 and Figure 4B. Mainly include \#1 randomized controlled trial ( $\mathrm{S}$ value $=0.876>0.7$, embodies 18 authors), 

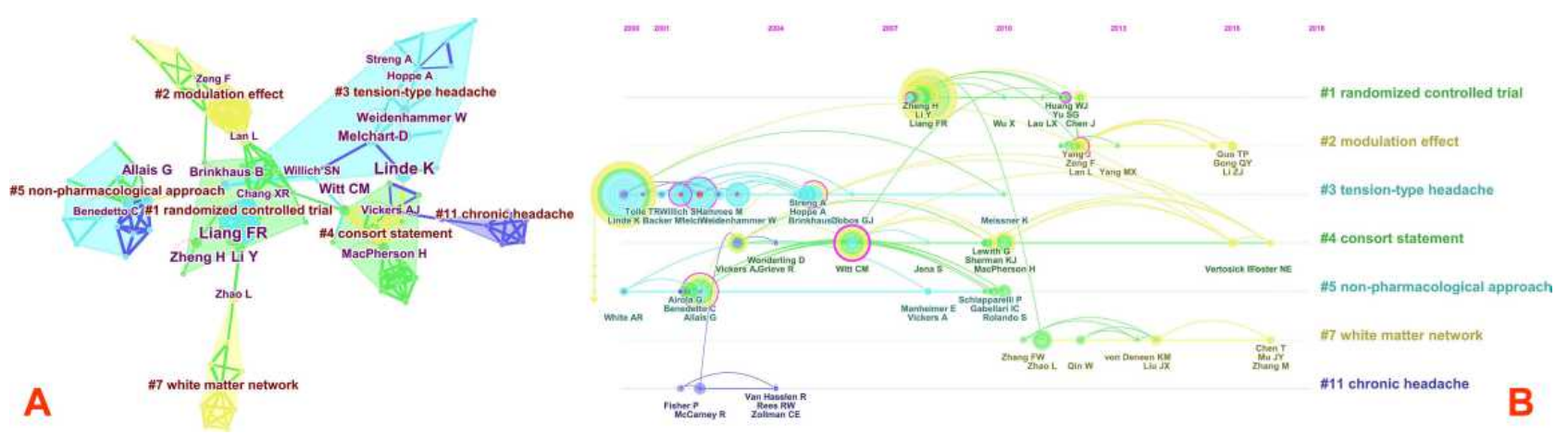

Figure 4 (A) Cluster map of authors based on label clusters with title terms. The combination of symbols and numbers delegates the authors' study of similar categories. (B) Timeline zone of authors on acupuncture for Migraine.

\#2 modulation effect ( $\mathrm{S}$ value $=0.974>0.7$, embodies 18 authors), $\# 4$ consort statement ( $\mathrm{S}$ value $=0.958>0.7$, embodies 16 authors), \#5 non-pharmacological approach (S value $=0.995>0.7$, embodies 14 authors), \#7 white matter network ( $\mathrm{S}$ value $=0.921>0.7$, embodies 9 authors), \#11 chronic headache (S value $=0.928>0.7$, embodies 6 authors). Then the following categories are the specific analysis of the same category of the authors. (1)Liang FR, Li Y, Sun GJ, and other authors' research on acupuncture for migraine can be classified into the same category, namely: randomized controlled trial (\#1). And Li Y is the representative researcher in this category. Li Y et al ${ }^{27,28}$ observed the effect of acupuncture for migraines through a multicenter randomized controlled trial. 480 migraine patients were randomly divided into 4 groups (Shaoyangspecific acupuncture, Shaoyang-nonspecific acupuncture, Yangming-specific acupuncture, or sham acupuncture control). Each group adopted a different treatment plan. It was found that acupuncture was more effective than sham acupuncture for almost all secondary results, and there was little correlation between acupuncture methods and results. Shaoyang specific acupuncture points did not produce better results than other acupuncture points, indicating that the role of specific acupuncture points plays a small role in the overall effect, and non-specific effects (such as expected and patient-doctor interaction) may be more prominent, so the research results may not be extended to Western populations. And it provides a direction for follow-up researchers to study acupuncture for migraines from the non-specific effects of acupuncture as an entry point. (2)The authors engaged in \#2 modulation effect mainly include Li ZJ, Lan L, Yang J, etc. Authors such as $\mathrm{Li} \mathrm{Z}^{29}$ used a randomized controlled trial to compare the resting-state functional connection of midbrain periaqueductal gray (PAG) between patients with migraine without aura and healthy controls and observe how acupuncture treatment affects the resting-state functional connectivity of migraine patients without aura.

Table 5 Authors Engaged in Acupuncture for Migraine That Details of Knowledge Clusters

\begin{tabular}{|l|c|c|c|c|c|}
\hline Cluster ID & Size & Silhouette & Mean (Year) & Label (LLR) & Label (MI) \\
\hline 1 & 18 & 0.876 & 2009 & Randomized controlled trial & Economic analysis \\
\hline 2 & 17 & 0.974 & 2014 & Modulation effect & Economic analysis \\
\hline 3 & 17 & 0.883 & 2004 & Tension-type headache & Economic analysis \\
\hline 4 & 16 & 0.958 & 2009 & Consort statement & Economic analysis \\
\hline 5 & 14 & 0.995 & 2005 & Non-pharmacological approach & Economic analysis \\
\hline 7 & 9 & 0.921 & 2014 & White matter network & Economic analysis \\
\hline 11 & 6 & 0.928 & 2003 & Chronic headache & Clinical trial \\
\hline
\end{tabular}

Note: The cluster analysis results mainly include cluster ID, mean year, size, silhouette, label (LLR), and label (MI). Cluster ID is the number after clustering, and Size represents the number of members contained in the cluster. The larger the Size is, the smaller the number. Mean Year represents the average year of the literature in the cluster, which can be used to judge the distance of the cited literature in the cluster. The larger the log-likelihood ratio (LLR) is, the more representative the cluster category; mutual information (MI) is mainly used to represent the relationship between terms and categories in text mining, and it does not consider the frequency of feature words. 
Table 6 Cited Reference Contributed to Co-Citations on Acupuncture for Migraine from 2000 to 2019

\begin{tabular}{|c|c|c|c|c|}
\hline No. & Cited Reference & Frequency & Cited Reference & Centrality \\
\hline 1 & Linde K $(2005)^{32}$ & 104 & Karst M $(2001)^{45}$ & 0.80 \\
\hline 2 & Diener HC $(2006)^{33}$ & 95 & Carlsson C (2002) ${ }^{46}$ & 0.71 \\
\hline 3 & Linde K $(2009)^{34}$ & 53 & Xue CCL $(2004)^{47}$ & 0.71 \\
\hline 4 & $\mathrm{Li} Y(2012)^{28}$ & 45 & Coeytaux RR $(2005)^{48}$ & 0.68 \\
\hline 5 & Melchart D $(2005)^{35}$ & 41 & Manias P $(2000)^{49}$ & 0.61 \\
\hline 6 & Silberstein SD $(2004)^{36}$ & 40 & Wallasch TM $(2012)^{50}$ & 0.58 \\
\hline 7 & Wang LP $(20 \mathrm{II})^{37}$ & 38 & Alecrim-andrade J $(2008)^{51}$ & 0.50 \\
\hline 8 & Witt C $(2005)^{38}$ & 34 & Endres HG $(2007)^{52}$ & 0.49 \\
\hline 9 & Vickers AJ $(2012)^{39}$ & 34 & Yang CP $(2011)^{53}$ & 0.42 \\
\hline 10 & Scharf HP $(2006)^{40}$ & 33 & Ramsay DJ $(1998)^{54}$ & 0.38 \\
\hline II & Bes A $(2013)^{4 I}$ & 31 & Wang LP $(201 \mathrm{I})^{37}$ & 0.30 \\
\hline 12 & Vickers AJ $(2004)^{31}$ & 31 & Linde K $(2006)^{55}$ & 0.29 \\
\hline 13 & Brinkhaus B (2006) ${ }^{42}$ & 28 & Kaptchuk TJ $(2006)^{56}$ & 0.26 \\
\hline 14 & Allais G $(2002)^{43}$ & 28 & Melchart D (1999) ${ }^{44}$ & 0.24 \\
\hline 15 & Melchart D (1999) ${ }^{44}$ & 27 & Streng A $(2006)^{57}$ & 0.22 \\
\hline
\end{tabular}

Note: In this table, the other two identical columns represent different rankings. The left column of the table represents the Cited References that have published acupuncture for migraine. The frequency of contribution is sorted from high to low. The column on the right represents the sorting from high to low according to centrality.

Studies have shown that damage to the descending pain modulatory system of migraine patients can return to normal after effective acupuncture treatment. This supplies a basis for us to study acupuncture treatment of migraine with the help of functional magnetic resonance imaging technology. (3)Zhang M, Tian J, Liu JX, and other author concentrated on acupuncture for migraine can be classified into \#7 white matter network. The most critical researcher in this direction is Liu JX. Liu JX ${ }^{30}$ studied the topology of the white matter network in patients with migraines without aura and healthy controls through a double-blind randomized controlled trial. Then, patients with simple paroxysmal migraines without aura were randomly divided into a traditional acupuncture group and sham acupuncture group, focusing on the results of the sham acupuncture group to study the placebo response of migraine patients. The results show that the placebo response can be identified a priori in migraine patients and that the specific topological properties of the brain structure network are the basis of the clinical placebo effect. (4)The following authors are included in the \#11 chronic headache: Vickers AJ, Zollman CE, McCarney R. Among them, the most prominent research is Vickers AJ. Vickers $\mathrm{AJ}^{31}$ used a randomized controlled trial to explore the impact of the "use acupuncture" policy on headaches, health status, sick leave days, and resource utilization of chronic headache patients. Acupuncture not only reduced the number of headache days for migraine patients but also decreased the rate of drug use, the number of general practitioners, and the time spent on sick leave. It shows that acupuncture has sustained and clinically relevant benefits for primary care of chronic headaches, especially migraines.

\section{Analysis of Cited Reference}

The top 15 references in frequency and centrality are listed in Table 6. Among them, the top 5 references with the highest co-citations are Linde $\mathrm{K} \quad(2005)^{32}$ (Frequency=104), Diener HC (2006), ${ }^{33}$ Linde K (2009), ${ }^{34}$ Li Y (2012), ${ }^{28}$ and Melchart D (2005), ${ }^{35}$ and the highest centrality is Karst M $(2001)^{36,45}$ (Centrality $=0.80$ ) and is considered as the most important reference in the field, followed by Carlsson C (2002) ${ }^{37,46}$, Xue CCL $(2004)^{38,47}$, Coeytaux RR $(2005)^{39,48}$ and Manias P (2000). ${ }^{40,49}$ The co-citation map of references 

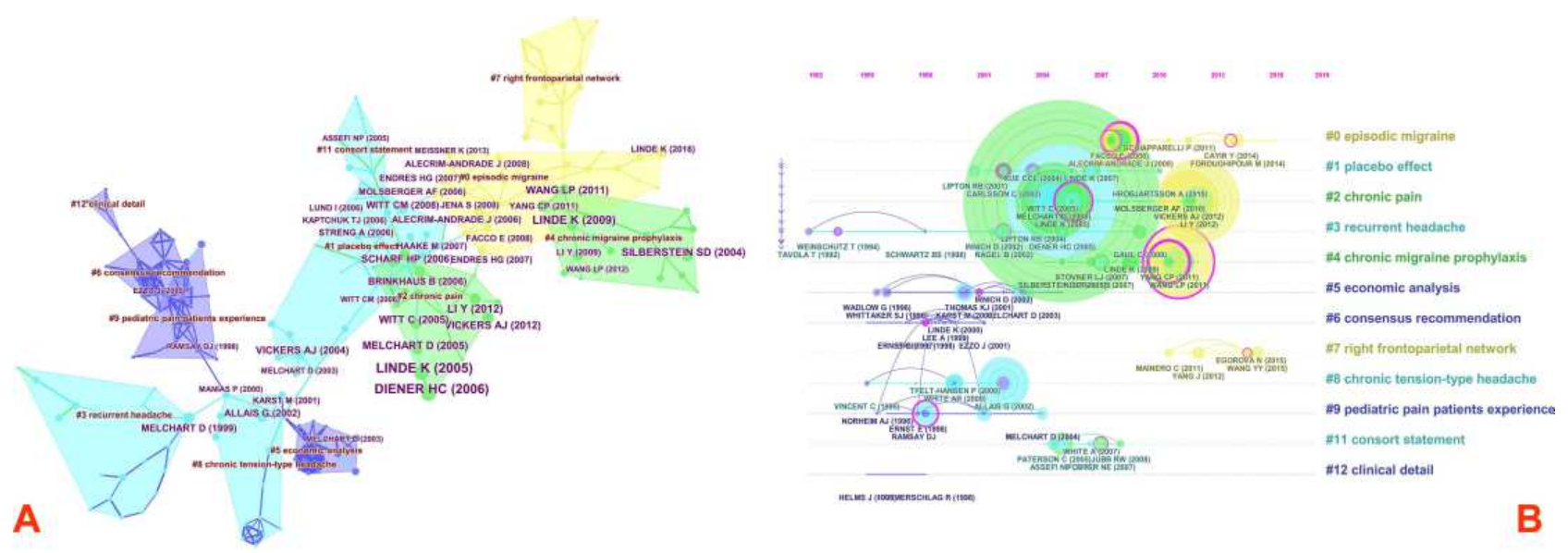

Figure 5 (A) Cluster map of Cited Reference based on label clusters with title terms. The combination of symbols and numbers delegates the Cited References' study of similar categories. (B) Timeline zone of Cited Reference on acupuncture for Migraine.

suggests the scientific relevance of the publications (Figure 5A). Here, the Modularity Q (0.8462) was higher than 0.7 , which indicates that the network was reasonable. All clusters were labeled with index terms extracted from the references. The timeline view for all clusters, which stated clearly the time span and research progress in the development and evolution of each cluster sub-domain is presented in Figure 5B. In the cluster map, the 12 largest clusters (Table 7) (small clusters were automatically filtered), included $\# 0$ episodic migraine
(Silhouette $=0.939>0.7$, Contains 27 references), \#1 placebo effect, \#2 chronic pain, \#3 recurrent headache, \#4 chronic migraine prophylaxis, \#5 economic analysis, \#6 consensus recommendation, \#7 right frontoparietal network, \#8 chronic tension-type headache, \#9 pediatric pain patients experience, \#11 consort statement, \#12 clinical detail. Among them, the research categories closely related to acupuncture for migraine are as follows: (1) \#0 episodic migraine: Linde $\mathrm{K}$ et $\mathrm{al}^{58}$ conducted a systematic review on the treatment of paroxysmal migraine with

Table 7 Cited Reference Concerned with Acupuncture for Migraine That Details of Knowledge Clusters

\begin{tabular}{|c|c|c|c|c|c|}
\hline Cluster ID & Size & Silhouette & Mean (Year) & Label (LLR) & Label (MI) \\
\hline 0 & 27 & 0.939 & 2011 & Episodic migraine & Prospective observational study \\
\hline I & 24 & 0.916 & 2005 & Placebo effect & Prospective observational study \\
\hline 2 & 23 & 0.895 & 2007 & Chronic pain & Prospective observational study \\
\hline 3 & 21 & 0.967 & 1999 & Recurrent headache & Prospective observational study \\
\hline 4 & 21 & 0.951 & 2008 & Chronic migraine prophylaxis & Prospective observational study \\
\hline 5 & 20 & 0.991 & 2000 & Economic analysis & Prospective observational study \\
\hline 6 & 18 & 0.864 & 1998 & Consensus recommendation & Prospective observational study \\
\hline 7 & 17 & 0.973 & 2013 & Right frontoparietal network & Prospective observational study \\
\hline 8 & 14 & 0.921 & 1999 & Chronic tension-type headache & Prospective observational study \\
\hline 9 & 14 & 0.893 & 1998 & Pediatric pain patients experience & Chronic pain \\
\hline II & 9 & 0.971 & 2006 & Consort statement & Prospective observational study \\
\hline 12 & 4 & 0.944 & 1996 & Clinical detail & Chronic pain \\
\hline
\end{tabular}

Note: The cluster analysis results mainly include cluster ID, mean year, size, silhouette, label (LLR), and label (MI). Cluster ID is the number after clustering, and Size represents the number of members contained in the cluster. The larger the Size is, the smaller the number. Mean Year represents the average year of the literature in the cluster, which can be used to judge the distance of the cited literature in the cluster. The larger the log-likelihood ratio (LLR) is, the more representative the cluster category; mutual information (MI) is mainly used to represent the relationship between terms and categories in text mining, and it does not consider the frequency of feature words. 
acupuncture and found that it is beneficial to add acupuncture in the treatment of acute migraine attacks or routine care; compared with fake acupuncture, compared with sham acupuncture, real acupuncture intervention is related to small and statistically significant effect after treatment and follow-up; paralleled with drug prevention, acupuncture has an advantage when treatment is completed. A series of randomized controlled trials subsequently demonstrated the above conclusions. Cayir $\mathrm{Y}^{59}$ analyzed the potential mechanism of acupuncture to relieve migraine from the perspective of the microscopic composition of blood, and found that it may be related to the reduction of matrix metalloproteinase-2 activity; Nevertheless, the focus of Enrico Facco 's study ${ }^{60}$ is to analyze the effectiveness of real acupuncture treatment in migraine without aura. The results demonstrate that compared with only using rizatriptan, real acupuncture is the only treatment that can provide a stable therapeutic effect. These studies are of great significance to acupuncture for migraine.(2) \#2 chronic pain: Vickers AJ's meta-analysis ${ }^{39}$ of individualized patient data through acupuncture treatment of chronic pain (back and neck pain, osteoarthritis, chronic headaches, and shoulder pain) found that acupuncture is effective in treating chronic pain, not just a placebo, and the conclusion of this analysis is somewhat different from some previous randomized controlled trials, which show that the treatment of migraine is not distinguished between patients who receive sham acupuncture, acupuncture or standard therapies; therefore, this suggests that follow-up researchers need to do more Large-sample, multi-center randomized controlled trials to further verify the effectiveness of acupuncture in the treatment of migraine and further clarify its treatment mechanism. (3)\#3 recurrent headache: Melchart ${ }^{4}$ Dused a systematic review to evaluate the effectiveness of acupuncture in the treatment of recurrent headaches. The existing evidence shows that acupuncture has a role in the treatment of recurrent headaches. However, the quality and quantity of evidence are not entirely convincing. Thus, large-scale clinical research is needed to evaluate the effectiveness and efficiency of acupuncture under real-life conditions. (4)\#7 right frontoparietal network: $\mathrm{Li} \mathrm{KS}$ analyzed the related effects of standard acupuncture on the right frontalparietal network of migraine patients based on functional magnetic resonance imaging technology. The results exhibited that acupuncture for migraine patients without aura is associated with the reduction of the inherent functional connection to the right frontal-parietal neural network. This provides new insights into the treatmentrelated neurological responses of patients with migraine without aura, and also offer a potential functional approach for treatment evaluation.

\section{Analysis of Keywords}

Table 8 shows keywords contributed to publications on acupuncture for migraine, the five most frequently used keywords were migraine (Frequency=234), acupuncture (228), headache (103), pain (81), and randomized controlled trial (78). And the most considerable keyword is needle acupuncture (centrality $=0.99$ ), followed by tension type headache (0.98), aura (0.84), prophylactic treatment $(0.71)$, and quality of life $(0.57)$. Ten clusters were obtained, with a Modularity Q of 0.7905. Mean Silhouette was $0.7733>0.7$. The 10 largest clusters are presented in Table 9. The timeline view shows that new keywords appeared almost every year (Figure 6B), and Figure 6A shows the Keyword-term cluster view, contained $\# 0$ topiramate treatment (Silhouette $=0.972>0.7$, Contains 17 keywords), \#1 consort statement, \#2 sham acupuncture intervention, \#3 further research, \#4 sham acupuncture intervention, \#5 episodic migraine, \#6 3-year follow-up study, \#7 prophylactic treatment, \#8 alternative therapy, \#9 new era. Among them, the research categories closely related to acupuncture for migraine are as follows: topiramate treatment: Yang $\mathrm{CP}^{53,62}$ compared the efficacy and tolerability of acupuncture and topiramate in the prevention of chronic migraine through a randomized controlled trial. The results showed that acupuncture is better than topiramate in the treatment of chronic migraine patients, mainly in the number of days of moderate/severe headache per month, the number of headache days per month, headache disability, several quality of life indicators reported by patients, and psychological stress. Hence, acupuncture should be considered as a treatment option for chronic migraine patients who are willing to receive this preventive treatment. sham acupuncture intervention: Foroughipour $\mathrm{M}^{63}$ evaluated the effect of acupuncture in routine migraine prevention measures through a randomized controlled trial. Migraine patients were divided into an acupuncture group and a sham acupuncture group. The results showed that the monthly frequency of migraine patients in the true acupuncture group ranged from 5.1 to 3.4 , and the monthly seizure frequency of the sham acupuncture group decreased from 5.0 to 4.4. The difference was significant, 
Table 8 Keyword Contributed to Publications on Acupuncture for Migraine from 2000 to 2019

\begin{tabular}{|c|c|c|c|c|}
\hline No. & Keyword & Frequency & Keyword & Centrality \\
\hline 1 & Migraine & 234 & Needle acupuncture & 0.99 \\
\hline 2 & Acupuncture & 228 & Tension type headache & 0.98 \\
\hline 3 & Headache & 103 & Aura & 0.84 \\
\hline 4 & Pain & 81 & Prophylactic treatment & 0.71 \\
\hline 5 & Randomized controlled trial & 78 & Quality of life & 0.57 \\
\hline 6 & Efficacy & 75 & Therapy & 0.54 \\
\hline 7 & Prophylaxi & 67 & Disability & 0.53 \\
\hline 8 & Placebo & 65 & Placebo & 0.50 \\
\hline 9 & Tension type headache & 62 & Double blind & 0.39 \\
\hline 10 & Double blind & 60 & Complementary medicine & 0.38 \\
\hline 11 & Low back pain & 46 & Electrical nerve stimulation & 0.30 \\
\hline 12 & Prevalence & 44 & Low back pain & 0.26 \\
\hline 13 & Multicenter & 41 & Primary care & 0.25 \\
\hline 14 & Clinical trial & 39 & Impact & 0.22 \\
\hline 15 & Electroacupuncture & 31 & Electroacupuncture & 0.21 \\
\hline 16 & Trial & 31 & Prevention & 0.21 \\
\hline 17 & Osteoarthriti & 30 & Topiramate & 0.21 \\
\hline 18 & Management & 25 & Reliability & 0.20 \\
\hline 19 & Migraine prophylaxi & 24 & Onabotulinumtoxina & 0.18 \\
\hline 20 & Knee & 24 & Migraine & 0.17 \\
\hline
\end{tabular}

Note: In this table, the other two identical columns represent different rankings. The left column of the table represents keywords that have published acupuncture for migraine. The frequency of contribution is sorted from high to low. The column on the right represents the sorting from high to low according to centrality.

suggesting that acupuncture can be seen as an auxiliary method of preventive drug treatment for patients with migraines, and preventive drug treatment cannot reduce the number of migraine attacks. Then Yang $\mathrm{Y}^{64}$ compared the efficacy of acupuncture and sham acupuncture for migraines through meta-analysis. Although current clinical evidence demonstrates that acupuncture is better than sham acupuncture in the treatment of migraines, it has a higher total effective rate and low recurrence rate. However, large-sample clinical randomized controlled trials are still needed for in-depth verification and analysis. prophylactic treatment: This category mainly involves two aspects of conventional acupuncture and ear acupuncture. Wallasch TM et $\mathrm{al}^{50}$ aims to use transcranial Doppler ultrasound to assess the effect of acupuncture on the cerebrovascular response of migraine patients. Conventional acupuncture is used. Acupuncture points include Hegu, Zusanli, Waiguan, Zulinqi, Houxi, Shenmai, Baihui, Fengchi, Taiyang, Taixi, Sizhukong, etc., and the results display that standardized acupuncture for migraine patients may have a positive effect on the dysfunction of the cerebrovascular autonomic nerve stimulation response, but it has no positive effect on the cerebrovascular tone at rest. It provides a methodological reference for us to study migraines from the autonomous cerebrovascular response. Next is the study of ear acupuncture to prevent chronic migraine, Allais $\mathrm{G}$ et $\mathrm{al}^{65}$ reveals that ear acupuncture can not only treat acute migraine but also supplies potential evidence for migraine prevention. Also, the 
Table 9 Keyword Related to Acupuncture for Migraine That Details of Knowledge Clusters

\begin{tabular}{|l|c|c|c|c|c|}
\hline Cluster ID & Size & Silhouette & $\begin{array}{c}\text { Mean } \\
\text { (Year) }\end{array}$ & Label (LLR) & Label (MI) \\
\hline 0 & 17 & 0.972 & 2005 & Topiramate treatment & Chronic daily headache \\
\hline 1 & 13 & 0.876 & 2005 & Consort statement & Chronic daily headache \\
\hline 2 & 13 & 0.964 & 2004 & Sham acupuncture intervention & Chronic daily headache \\
\hline 3 & 12 & 0.893 & 2007 & Further research & Chronic daily headache \\
\hline 4 & 12 & 0.718 & 2005 & Sham acupuncture intervention & Botulinum toxin type \\
\hline 5 & 12 & 0.870 & 2011 & Episodic migraine & Controlled trial \\
\hline 6 & 10 & 0.954 & 2005 & 3 -year follow-up study & controlled trial \\
\hline 7 & 8 & 0.646 & 2008 & Prophylactic treatment & Controlled trial \\
\hline 8 & 8 & 0.797 & 2001 & Alternative therapy & Controlled trial \\
\hline 9 & 10 & 2008 & New era & Controlled trial \\
\hline
\end{tabular}

Note: The cluster analysis results mainly include Cluster ID, mean year, size, silhouette, label (LLR), and label (MI). Cluster ID is the number after clustering, and Size represents the number of members contained in the cluster. The larger the Size is, the smaller the number. Mean Year represents the average year of the literature in the cluster, which can be used to judge the distance of the cited literature in the cluster. The larger the log-likelihood ratio (LLR) is, the more representative the cluster category; mutual information (MI) is mainly used to represent the relationship between terms and categories in text mining, and it does not consider the frequency of feature words.

therapeutic effect is significant during the extended period of once every 3 weeks. This prompts that more closely scheduling treatment may be of greater benefit. However, the limitation of this study is the lack of a control group, and the observation case is female. And it offers a basis for acupuncture at special parts to prevent migraines.

By comparing the results of institution clustering, author clustering, reference clustering, and keyword clustering, we found that randomized controlled trials of acupuncture prevention and treatment of migraine are common content, so it can be regarded as the key research content in the field.

\section{Conclusions}

A new perspective on the trends of acupuncture for migraine is provided by this study. Although this study has certain limitations, it fully promulgates the global trend of acupuncture for migraine and presents it to readers in the form of a visual knowledge mapping.
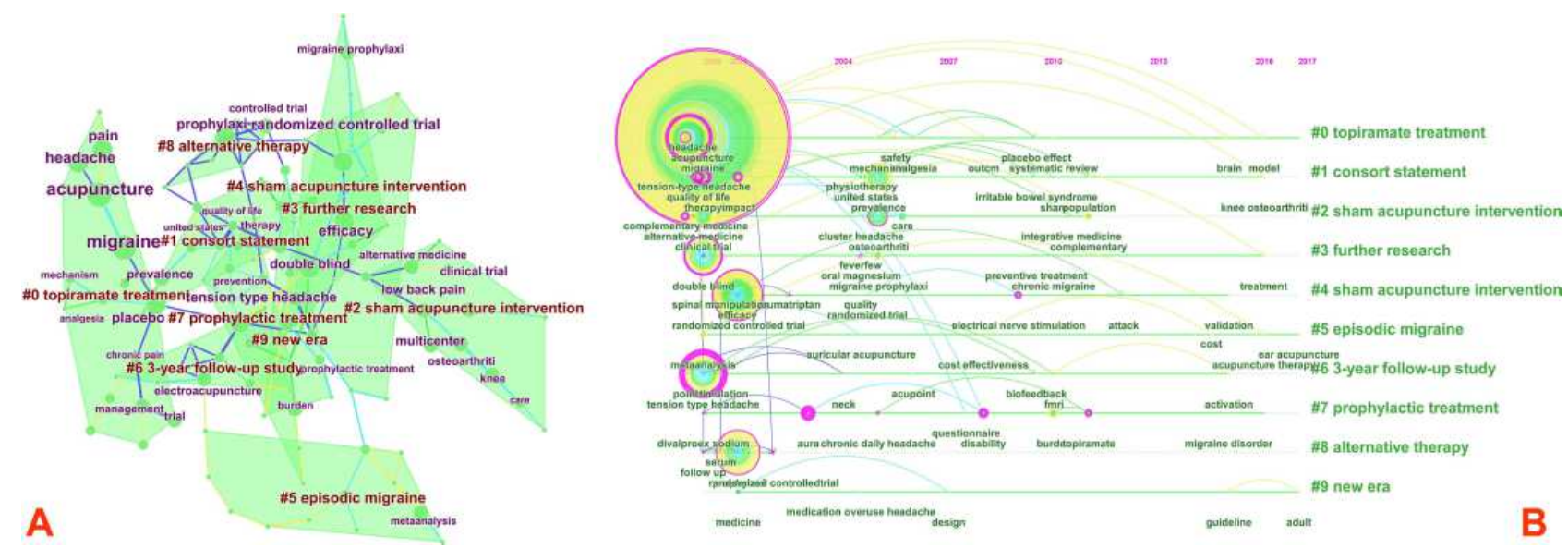

Figure 6 (A) Cluster map of Keyword based on label clusters with title terms. The combination of symbols and numbers delegates the Keywords' study of similar categories. (B) Timeline zone of Keyword on acupuncture for Migraine. 
Most articles in this field were published in the USA and China, with the Technical University of Munich and Beijing University of Chinese Medicine contributing the most publications. The author who publishes the most papers is Linde $\mathrm{K}$, and the most frequently cocited literature is Linde K (2005). Most of the countries engaged in this field are in developed countries, and most of the momentous institutions are in China. However, the cooperation between different institutions and international cooperation is relatively weak. And the vital reference is Karst M published in 2001. Moreover, through knowledge mapping analysis, it is found that Randomized controlled trials of acupuncture in the prevention and treatment of migraine are the most important research content in this field. At last, the results of this research may offer researchers useful information, such as research frontiers, potential collaborators, Countries, and cooperative institutions.

\section{Data Sharing Statement}

The raw data supporting the conclusions of this article will be made available by Yanqing Zhao and Li Huang, without undue reservation.

\section{Author Contributions}

YZ designed this study. LH performed the search. YZ collected data. LH and WL rechecked data. YZ and LH performed analysis. HG and ML: critically revised the work. $\mathrm{YZ}$ and LH have contributed equally to this work and should be considered co-first authors. All authors made substantial contributions to conception and design, acquisition of data, or analysis and interpretation of data; took part in drafting the article or revising it critically for important intellectual content; agreed to submit to the current journal; gave final approval of the version to be published; and agree to be accountable for all aspects of the work.

\section{Research Limitations}

There are several limitations of this study. Firstly, although the search strategy searches for synonyms of the MeSH subject words in PubMed, it may still cause some works of literature to be missed. Secondly, in our research, we only use the Science Citation Index-Expanded (SCIE) module in the Web of Science database. Although it contains most of the literature needed for research, it may still cause the loss of some literature. Thirdly, Citespace's function of removing duplication has certain limitations, which may bias the research results.

\section{Funding}

This study was supported by grants from the Shanghai Science and Technology Commission, "science and technology innovation action plan" clinical medicine project (no. 18401971200), Shanghai 13th five years plan key clinical specialty construction project (no. shslczdzk04901). Scientific research project of Putuo Hospital, Shanghai University of Traditional Chinese Medicine (no. 2020304A). Budget Project of Shanghai University of Traditional Chinese Medicine (no. 2019WK118).

\section{Disclosure}

The authors report no conflicts of interest in this work.

\section{References}

1. Gazerani P. Migraine and Diet. Nutrients. 2020;12.

2. Pelzer N, Louter MA, van Zwet EW, et al. Linking migraine frequency with family history of migraine. Cephalalgia. 2019;39:229-236.

3. Burch RC, Buse DC, Lipton RB. Migraine: epidemiology, burden, and comorbidity. Neurol Clin. 2019;37:631-649.

4. Lagman-Bartolome AM, Lay C. Migraine in Women. Neurol Clin. 2019;37:835-845.

5. Youssef PE, Mack KJ. Episodic and chronic migraine in children. Dev Med Child Neurol. 2020;62:34-41.

6. Wijeratne T, Tang HM, Crewther D, et al. Prevalence of migraine in the elderly: a narrated review. Neuroepidemiology. 2019;52:104-110.

7. Parikh SK, Young WB. Migraine: stigma in Society. Curr Pain Headache Rep. 2019;23:8.

8. Silberstein SD, Lee L, Gandhi K, et al. Health care resource utilization and migraine disability along the migraine continuum among patients treated for migraine. Headache. 2018;58:1579-1592.

9. Yang X, Zhang J, Li N, et al. Progress in drug prevention and treatment of migraine. Chin $J$ Clin Pharmacol Ther. 2020;25:1429-1435.

10. Gonzalez-Hernandez A, Marichal-Cancino BA, MaassenVanDenBrink A, et al. Side effects associated with current and prospective antimigraine pharmacotherapies. Expert Opin Drug Metab Toxicol. 2018;14:25-41.

11. Munksgaard SB, Madsen SK, Wienecke T. Treatment of medication overuse headache-A review. ACTA NEUROL SCAND. 2019;139:405-414.

12. Ou MQ, Fan WH, Sun FR, et al. A systematic review and meta-analysis of the therapeutic effect of acupuncture on migraine. Front Neurol. 2020;11:596.

13. Urits I, Patel M, Putz ME, et al. Acupuncture and its role in the treatment of migraine headaches. Neurol Ther. 2020;9:375-394.

14. Xu Z-M, Min J, Xiao L, et al. [Clinical practice guideline for migraine with traditional Chinese medicine(draft version for comments)]. Zhongguo Zhong Yao Za Zhi. 2020;45:5057-5067. Chinese.

15. Zhou S, Tao Z, Zhu Y, et al. Mapping theme trends and recognizing hot spots in postmenopausal osteoporosis research: a bibliometric analysis. PEERJ. 2019;7:e8145.

16. Tao L, Zhou S, Tao Z, et al. The publication trends and hot spots of scoliosis research from 2009 to 2018: a 10-year bibliometric analysis. Ann Transl Med. 2020;8:365.

17. Li S, Wang H, Zheng H, et al. Bibliometric Analysis of Pediatric Liver Transplantation Research in PubMed from 2014 to 2018. Med Sci Monit. 2020;26:e922517. 
18. Zhu X, Niu X, Li T, et al. Identification of research trends concerning application of stent implantation in the treatment of pancreatic diseases by quantitative and biclustering analysis: a bibliometric analysis. PEERJ. 2019; 7:e7674.

19. Zheng K, Wang X. Publications on the association between cognitive function and pain from 2000 to 2018: a bibliometric analysis using citespace. Med Sci Monit. 2019;25:8940-8951.

20. Qi B, Jin S, Qian H, et al. Bibliometric analysis of chronic traumatic encephalopathy research from 1999 to 2019. Int J Environ Res Public Health. 2020;17.

21. Mao Z, Liu C, Chen S, et al. A bibliometric analysis of exertional heat stroke research in Web of Science. Mil Med Res. 2016;3:31.

22. Huang L, Zhao Y, Xiang M. Knowledge mapping of acupuncture for cancer pain: a scientometric analysis (2000-2019). J Pain Res. 2021;14:343-358.

23. Ke L, Lu C, Shen R, et al. Knowledge mapping of drug-induced liver injury: a scientometric investigation (2010-2019). Front Pharmacol. 2020;11:842.

24. Wang SQ, Gao YQ, Zhang C, et al. A Bibliometric analysis using citespace of publications from 1999 to 2018 on patient rehabilitation after total knee arthroplasty. Med Sci Monit. 2020;26:e920795.

25. Miao Y, Liu R, Pu Y, et al. Trends in esophageal and esophagogastric junction cancer research from 2007 to 2016: a bibliometric analysis. Medicine (Baltimore). 2017;96:e6924.

26. Fu J, Zhang X, Zhao Y, et al. Scientific production and citation impact: a bibliometric analysis in acupuncture over three decades. Scientometrics. 2012;93:1061-1079.

27. Li Y, Liang F, Yu S, et al. Randomized controlled trial to treat migraine with acupuncture: design and protocol. Trials. 2008;9:57.

28. Li Y, Zheng H, Witt CM, et al. Acupuncture for migraine prophylaxis: a randomized controlled trial. CMAJ. 2012;184:401-410.

29. Li Z, Liu M, Lan L, et al. Altered periaqueductal gray resting state functional connectivity in migraine and the modulation effect of treatment. Sci Rep. 2016;6:20298.

30. Liu J, Ma S, Mu J, et al. Integration of white matter network is associated with interindividual differences in psychologically mediated placebo response in migraine patients. Hum Brain Mapp. 2017;38:5250-5259.

31. Vickers AJ, Rees RW, Zollman CE, et al. Acupuncture for chronic headache in primary care: large, pragmatic, randomised trial. $B M J$ 2004;328:744.

32. Linde K, Streng A, Jurgens S, et al. Acupuncture for patients with migraine: a randomized controlled trial. JAMA. 2005;293:2118-2125.

33. Diener HC, Kronfeld K, Boewing G, et al. Efficacy of acupuncture for the prophylaxis of migraine: a multicentre randomised controlled clinical trial. Lancet Neurol. 2006;5:310-316.

34. Linde K, Allais G, Brinkhaus B, et al. Acupuncture for migraine prophylaxis. Cochrane Database Syst Rev. 2009;D1218.

35. Melchart D, Streng A, Hoppe A, et al. Acupuncture in patients with tension-type headache: randomised controlled trial. BMJ. 2005;331:376-382.

36. Silberstein SD. Migraine pathophysiology and its clinical implications. Cephalalgia. 2004;24(Suppl 2):2-7.

37. Wang LP, Zhang XZ, Guo J, et al. Efficacy of acupuncture for migraine prophylaxis: a single-blinded, double-dummy, randomized controlled trial. Pain. 2011;152:1864-1871.

38. Witt C, Brinkhaus B, Jena S, et al. Acupuncture in patients with osteoarthritis of the knee: a randomised trial. Lancet. 2005;366:136-143.

39. Vickers AJ, Cronin AM, Maschino AC, et al. Acupuncture for chronic pain: individual patient data meta-analysis. Arch Intern Med. 2012;172:1444-1453.

40. Scharf HP, Mansmann U, Streitberger K, et al. Acupuncture and knee osteoarthritis: a three-armed randomized trial. Ann Intern Med. 2006;145:12-20.
41. The International Classification of Headache Disorders, 3rd edition (beta version). Cephalalgia. 2013;33:629-808.

42. Brinkhaus B, Witt CM, Jena S, et al. Acupuncture in patients with chronic low back pain: a randomized controlled trial. Arch Intern Med. 2006;166:450-457.

43. Allais G, De Lorenzo C, Quirico PE, et al. Acupuncture in the prophylactic treatment of migraine without aura: a comparison with flunarizine. Headache. 2002;42:855-861.

44. Melchart D, Linde K, Fischer P, et al. Acupuncture for recurrent headaches: a systematic review of randomized controlled trials. Cephalalgia. 1999;19(779-86):765.

45. Karst M, Reinhard M, Thum P, et al. Needle acupuncture in tension-type headache: a randomized, placebo-controlled study. Cephalalgia. 2001;21:637-642.

46. Carlsson C. Acupuncture mechanisms for clinically relevant long-term effects--reconsideration and a hypothesis. Acupunct Med. 2002;20:82-99.

47. Xue CC, Dong L, Polus B, et al. Electroacupuncture for tension-type headache on distal acupoints only: a randomized, controlled, crossover trial. Headache. 2004;44:333-341.

48. Coeytaux RR, Kaufman JS, Kaptchuk TJ, et al. A randomized, controlled trial of acupuncture for chronic daily headache. Headache. 2005;45:1113-1123.

49. Manias P, Tagaris G, Karageorgiou K. Acupuncture in headache: a critical review. Clin J Pain. 2000;16:334-339.

50. Wallasch TM, Weinschuetz T, Mueller B, et al. Cerebrovascular response in migraineurs during prophylactic treatment with acupuncture: a randomized controlled trial. J Altern Complement Med. 2012;18:777-783.

51. Alecrim-Andrade J, Maciel-Junior JA, Carne X, et al. Acupuncture in migraine prevention: a randomized sham controlled study with 6-months posttreatment follow-up. Clin J Pain. 2008;24:98-105.

52. Endres HG, Diener HC, Molsberger A. Role of acupuncture in the treatment of migraine. Expert Rev Neurother. 2007;7:1121-1134.

53. Yang CP, Chang MH, Liu PE, et al. Acupuncture versus topiramate in chronic migraine prophylaxis: a randomized clinical trial. Cephalalgia. 2011;31:1510-1521.

54. Ramsay D, Bowman M, Greenman P, et al. Acupuncture. JAMA. 1998;280:1518-1524.

55. Linde K, Streng A, Hoppe A, et al. Treatment in a randomized multicenter trial of acupuncture for migraine (ART migraine). Forsch Komplementmed. 2006;13:101-108.

56. Kaptchuk TJ, Stason WB, Davis RB, et al. Sham device v inert pill: randomised controlled trial of two placebo treatments. BMJ. 2006;332:391-397.

57. Streng A, Linde K, Hoppe A, et al. Effectiveness and tolerability of acupuncture compared with metoprolol in migraine prophylaxis. Headache. 2006;46:1492-1502.

58. Linde K, Allais G, Brinkhaus B, et al. Acupuncture for the prevention of episodic migraine. Cochrane Database Syst Rev. 2016;D1218.

59. Cayir Y, Ozdemir G, Celik M, et al. Acupuncture decreases matrix metalloproteinase-2 activity in patients with migraine. Acupunct Med. 2014;32:376-380.

60. Facco E, Liguori A, Petti F, et al. Traditional acupuncture in migraine: a controlled, randomized study. Headache. 2008;48:398-407.

61. Li K, Zhang Y, Ning Y, et al. The effects of acupuncture treatment on the right frontoparietal network in migraine without aura patients. $J$ Headache Pain. 2015;16:518.

62. Yang CP, Chang MH, Li TC, et al. Predicting prognostic factors in a randomized controlled trial of acupuncture versus topiramate treatment in patients with chronic migraine. Clin $J$ Pain. 2013;29:982-987.

63. Foroughipour M, Golchian AR, Kalhor M, et al. A sham-controlled trial of acupuncture as an adjunct in migraine prophylaxis. Acupunct Med. 2014;32:12-16. 
64. Yang Y, Que Q, Ye X, et al. Verum versus sham manual acupuncture for migraine: a systematic review of randomised controlled trials. Acupunct Med. 2016;34:76-83.
65. Allais G, Sinigaglia S, Airola G, et al. Ear acupuncture in the prophylactic treatment of chronic migraine. NEUROL SCI. 2019;40:211-212.

\section{Publish your work in this journal}

The Journal of Pain Research is an international, peer reviewed, open access, online journal that welcomes laboratory and clinical findings in the fields of pain research and the prevention and management of pain. Original research, reviews, symposium reports, hypothesis formation and commentaries are all considered for publication. The manuscript management system is completely online and includes a very quick and fair peer-review system, which is all easy to use. Visit http:// www.dovepress.com/testimonials.php to read real quotes from published authors. 